\title{
Propane Gas Thermoelectric Generator with Microcontroller Integrated Development Environment Hot Side Temperature Control
}

\author{
Francis Onoroh ${ }^{1, *}$, Adeyinka Abdulquadri Oluwo ${ }^{2}$, Larry Orobome Agberegha ${ }^{3}$ \\ Full Affiliation, Department of Mechanical Engineering, University of Lagos, Akoka, Yaba, Lagos, Nigeria \\ Full Affiliation, Department of Mechanical Engineering, University of Lagos, Akoka, Yaba, Lagos, Nigeria \\ Full Affiliation, Department of Mechanical Engineering, Federal University of Petroleum Resources Effurun, Delta state, Nigeria
}

\begin{abstract}
A thermoelectric generator consists of various thermocouples arranged in series. A voltage is generated when a temperature gradient exists across the junctions of the thermocouples. This research modelled, developed and tested a thermoelectric generator with hot side temperature control using propane gas, in a microcontroller integrated development environment to protect the modules from excessive high temperatures. The models were solved using MAT$L A B$ for pictorial representation of the performance of the generator with temperature gradient, hot and cold side temperatures. The thermoelectric generator was developed using ten SP-184827145-SA modules. The temperature control circuitry consists of an ATMEGA32 microcontroller, DS18B30 temperature sensors, stepper motor, computer interface and power supply unit whose sole aim is to regulate the gas supply and hence control the flame temperature. The maximum open circuit voltage gotten was $25 \mathrm{~V}$ at 300 secs with hot side temperature of $120^{\circ} \mathrm{C}$ and cold side temperature $30^{\circ} \mathrm{C}$, with an efficiency of $3.6 \%$. It was also found out that the higher the temperature gradient the higher the voltage produced.
\end{abstract}

Keywords: Temperature gradient; Thermocouple; Flame temperature; ATMEGA32 microcontroller; Voltage.

\section{Introduction}

Thermoelectric generators are regarded as solid-state devices as they implore the use of Seebeck effect in its operation as it takes advantage of temperature difference to create electrical energy [1]. It can also be regarded as a heat engine as it converts heat energy to electrical energy. The basic unit of the thermoelectric generator is the thermoelectric modules which consist of several thermoelectric couple junctions connected electrically in series and thermally in parallel [2]. These modules when used within the required temperature range can be used to power basic domestic appliances and reduce the over dependence on the national grid. They are known to be noiseless in operation as they contain no moving parts, require little or no maintenance and are highly reliable with a long-life span [3], other advantages are their small sizes, simple mode of operation and they are environmentally friendly [1]. In order to safeguard and prolong the lifespan of the thermocouples temperature control measures must be adopted. Thermoelectric power systems find wide applications in medicine, telecommunications, gas pipelines and remote terrestrial applications; space probes like Mars Curiosity Rover and the efficiency of a vehicle can be enhanced, [4], [5].

A number of literatures exist in the field of thermoelectricity. Rohit et al [6] reported on the performance study of thermoelectric generator. They employed bismuth tellu- 
ride based thermoelectric modules and aluminium material in the design of the heat sink and an electric heater from an emissivity apparatus served as a heat source. Experimental temperature varied from $35^{\circ} \mathrm{C}$ to $150^{\circ} \mathrm{C}$ and from results obtained, it was reported that power generated was directly proportional to temperature on the hot surface. Ming et al [7] studied the influence of non-uniform high heat flux on thermal stress of thermoelectric power generator. They modelled mathematically the effect of heating uniformity on modules stress level using finite element analysis method and were able to obtain the most likely crack zone for different heat flux concentrations rates. They discovered that an increase in heat flow to the modules increases its efficiency but raises the temperature and thermal stresses of the modules. Ahiska and Dislitas [8] worked on a microcontroller based thermoelectric generator application. The generator was designed to function using 12 thermoelectric modules operating at a maximum temperature of $130^{\circ} \mathrm{C}$ and employed geothermal water as the heat source. A PIC16F877 Microcontroller programmed using Parsic program was used for the control of the system.

Nuwayhid et al [9] studied the development and testing of a domestic woodstove thermoelectric generator with natural convection cooling. A temperature map on common rural woodstoves was drawn to help determine the most favourable location for the thermoelectric modules to be placed. A maximum power of $4.2 \mathrm{~W}$ was obtained when a single module was used. They concluded that an increase in the number of modules without a corresponding increase in the heat source led to a decrease in electrical power generated. Chasmar and Stratton [10] studied the Thermoelectric Figure of Merit and its relation to thermoelectric generators. They related the popular criteria for evaluating module properties, the thermoelectric figure of merit with thermoelectric generators. They numerically evaluated the expression for the figure of merit of a semi-conductor of a given carrier mobility and lattice thermal conductivity. They presented graphically expression to find maximum figure of merit.

Singh et al [3] studied the development and investigation of a non-catalytic self-aspirating mesoscale premixed burner integrated thermoelectric power generator. The research was divided into two phases with the first phase concerned with optimization of the burner design in order to achieve a stable enclosed premixed flame and from the results obtained it could be concluded that the introduction of a premix zone and backward facing step in the gas burner design enhanced the mixing of reactants (propane and oxygen) thereby improving combustion and overall temperature. Also, the burner design proved that the addition of secondary air supply perpendicular to the stream had a significant effect on flame stabilization and clean combustion inside the burner. Although combustion was improved due to the burner designs adopted the amount of air needed or used for complete combustion couldn't be accounted for as it was a self-aspirating burner. The burner was designed for a maximum thermal output of $250 \mathrm{~W}$ as it accommodated two modules. Mishra et al [11] designed a low cost, easy to use thermoelectric generator that provides emergency electrical energy if there is power cut. The generator utilizes waste heat produce from silencers and combustors as input energy source. A thermoelectric module was used for the power generation purpose with the application of a temperature difference across the module. They concluded that power loss can be reduced by thermally insulating the modules.

Elghool et al [12] made a review on heat sink for thermo-electric power generation: classifications and parameters affecting performance. Heat sinks were classified into various categories such as passive, semi-active, active, liquid cooling and phase change cooling. Different types of heat sinks such as metal plate, fin heat sink, heat pipes and fan-fin heat sinks, liquid cooled plate, and microchannel were also reviewed. It was concluded that the efficiency of thermoelectric generators highly depends on good selection and careful designing of heat sink. Lee et al [13] worked on thermoelectric characterization and power generation using a silicon-on-insulator substrate to demonstrate techniques for measuring thermoelectric voltages and generating on-chip power. Lateral heat conduction in the overlayer of the silicon was used to establish temperature differentials, that reduces microfabrication complexity, compared to free-standing membranes, the siliconon-insulator based substrates may achieve smaller temperature gradient but are mechanically much more stable and do not require under etching.

Aranvindkaruppaiah et al [14] fabricated and analysed a thermoelectric generator for power generation. The scope was to design a thermoelectric 
generator (TEG) which produces power from exhaust waste heat in internal combustion (IC) engines (automobiles) to analyse the TEG materials used and also studied heat transfer in TEG using ANSYS. They concluded that TEG system of charging the battery could reduce the fuel consumption and also battery life used in automobiles could be increased. Jfri et al [15] used waste heat for thermoelectric-based energy harvesting that convert heat energy directly to electrical energy. They found out that bismuth telluride $\left(\mathrm{Bi}_{2} \mathrm{Te}_{3}\right)$ thermoelectric module is the most favourable material to produce more power and the voltage output is a function of temperature gradient across the modules.

Lay-Ekuakille et al [16] modeled and design a thermoelectric generator that extracts energy from human tissue and an array of sensors based on thermocouple effect. Specific conditioning circuit has been designed and implemented to supply biomedical hearing aid. Shah et al [5] analysed power generation through thermoelectric generators. Their research focuses on the use of waste heat energy to generate power through thermoelectric generators. The heat source flows across the sink of the TEG and converts it into electric energy. The result gotten shows that the efficiency of TEG's decreases as the size of the power plant increases. Stecanella et al [17] studied electricity generation using thermoelectric generators. The purpose of their research was to present an electricity generation plant using thermoelectric generator which recovers the thermal energy dissipated by combustion gases of an electric generator. The temperature values were obtained through thermal imager and type $\mathrm{K}$ laser thermocouples and as the temperature increases the load also increases. This present research, designed and developed a propane gas powered thermoelectric generator with ATMEGA 32 microcontroller using DS18B20 temperature sensors and 200 steps stepper motor for real time temperature regulation, the experimental setup consists of the thermoelectric generator, charge controller, storage batteries and a power inverter.

\section{Thermoelectric Performance Models}

According to Thomas Johann Seebeck, the specific voltage output of a thermoelectric couple is a function of temperature gradient and the Seebeck coefficient. Mathematically it is obtained from the relation [17]:

$$
v=\alpha\left(T_{h}-T_{c}\right)
$$

Where $\alpha$ equals Seebeck coefficient, $V / K, T h$ and $T_{c}$ are the hot side and cold side module temperatures respectively, ${ }^{\circ} \mathrm{C}$. For $\mathrm{N}$ number of modules with $n$ thermocouple junctions, the total voltage generated is obtained from:

$V=n N \alpha\left(T_{h}-T_{c}\right)$

The current generated by thermoelectric modules obtained directly from Ohm's law is:

$I=\frac{V}{R_{T}}$

Where $R_{T}$ is the sum of load and internal resistances of the thermoelectric materials, $\boldsymbol{\Omega}$. The load resistance to the internal resistances, $m$, is expressed as:

$m=\frac{R_{L}}{R}$

Where $R_{L}$ is load resistance, $\Omega, R$ is internal resistance of the module, $\Omega$. In the light of equation (4), the total resistance of the thermoelectric generator is expressed as:

$R_{T}=R(m+1)$

In the light of equation (2) and equation (5), equation (3) becomes:

$I=\frac{n N \alpha\left(T_{h}-T_{c}\right)}{R(m+1)}$

The internal resistance of the module is obtained from the relation:

$R=\frac{V_{\max }}{I_{\max }}$

Where $V_{\max }, V$ and $I_{\max }, A$, are the maximum module voltage and current respectively. Substituting equation (7) into equation (6), gives the output current from the thermoelectric module as:

$I=\frac{n N \alpha\left(T_{h}-T_{c}\right) I_{\max }}{V_{\max }(m+1)}$

Where $V_{\max }$ and $I_{\max }$ are obtained from the module manufacturer data sheet. The power output associated with the load resistance $R \mathrm{~L}$ is defined by the expression [4]: 
$P=I^{2} R_{L}$

In the light of equation (4) and equation (8), the power output expression is obtained as:

$$
P=\frac{m}{(m+1)^{2}}\left[\left(n N \alpha\left(T_{h}-T_{c}\right)\right)^{2}\right] \frac{I_{\max }}{V_{\max }}
$$

The heat supplied to the hot junction of the module is the sum of heat due to conduction, Peltier's and Joule's [18]:

$Q_{h}=Q_{k}+Q_{p}-Q_{j}$

Where $Q_{k}$ heat flow due to conduction, $W, Q_{p}$ heat flow due to Peltier effect, $W$, and $Q_{j}$ is Joule heating effect, $W$. The heat due to conduction is expressed as:

$Q_{k}=K\left(T_{h}-T_{c}\right)$

The thermal conductance, $\mathrm{W} / \mathrm{cm}^{2} \mathrm{~K}$, is obtained using the relation:

$K=\frac{k A}{L}$

Where $k$ is thermal conductivity, W/cmK, $A$ is the area, $\mathrm{cm}^{2}$, and $L, \mathrm{~cm}$ is the length of the thermoelectric module respectively. The Peltier heat is defined by the relation:

$Q_{p}=\alpha I T_{h}$

The Joule heat effect is defined as:

$Q_{j}=\frac{1}{2} I^{2} R$

Substituting equation (12), equation (14) and equation (15) into equation (11) gives the heat supplied by the heat source to the thermoelectric generator as:

$Q_{h}=\alpha I T_{h}+K\left(T_{h}-T_{c}\right)-\frac{1}{2} I^{2} R$

The source of heat to the thermoelectric generator is by combustion of propane gas in a bottled cylinder. The thermochemical equation for the combustion of propane is:

$\mathrm{C}_{3} \mathrm{H}_{8}+5 \mathrm{O}_{2}+18.81 \mathrm{~N}_{2} \rightarrow 3 \mathrm{CO}_{2}+4 \mathrm{H}_{2} \mathrm{O}+18.81 \mathrm{~N}_{2}$
The correlation for determination of the laminar flame speed is given as [19]:

$$
S_{L}=S_{L, r e f}\left(\frac{T_{o}}{T_{o, r e f}}\right)^{\gamma}\left(\frac{P}{P_{\text {ref }}}\right)^{\beta}\left(1-2.1 Y_{\text {dil }}\right)
$$

Where $T_{o, r e f}$ is the reference condition for gas temperature, $K, P_{r e f}$ is the reference condition for pressure, $\mathrm{kPa}, Y_{\text {dil }}$ is the mass fraction of diluent in the air-fuel mixture, $\gamma$ and $\beta$ temperature and pressure exponent respectively and $\phi$ is the Equivalent ratio. The reference laminar speed, and the pressure exponents are defined by equations:

$$
\begin{aligned}
& S_{L, r e f}=B_{M}+B_{2}\left(\phi-\varnothing_{M}\right)^{2} \\
& \gamma=2.18-0.8(\phi-1) \\
& \beta=-0.16+0.22(\phi-1)
\end{aligned}
$$

The constants $B_{M}, B_{2}$ and $\varnothing_{M}$ depend on fuel type. For propane gas their numerical values are; $34.22 \mathrm{~cm} / \mathrm{s},-138.65 \mathrm{~cm} / \mathrm{s}$ and 1.08 respectively [19]. The heat rejected at the cold junction of the module is given as:

$Q_{c}=\alpha I T_{c}+K\left(T_{h}-T_{c}\right)+\frac{1}{2} I^{2} R$

The heat sink attached to the thermoelectric modules is a water cooled chamber with five pass$\mathrm{es}$, the quantity of heat dissipated at the heat sink is computed as:

$Q_{c}=\rho \dot{V} c_{p}\left(T_{e}-T_{i}\right)$

Where $\rho$ is the density of water, $\mathrm{kg} / \mathrm{m}^{3}, \dot{V}$ flow rate of water, $\mathrm{m}^{3} / \mathrm{s}, T_{e}$ and $T_{i}$ are exit and inlet temperature of cooling water, ${ }^{\circ} \mathrm{C}, c_{p}$ is specific heat of water at constant pressure, $\mathrm{J} / \mathrm{kg} . \mathrm{K}$.

Efficiency of the thermoelectric generator is defined as the ratio of power output to that of the heat input:

$\eta=\frac{P}{Q_{h}}$

The efficiency can be expressed in the form:

$\eta=\eta_{c} \times \eta^{*}$

$\eta=\frac{T_{h}-T_{c}}{T_{h}} \eta^{*}$ 
Where $\eta^{*}$ depends on the module material, geometry and load on the generator and $\eta_{c}$ is Carnot efficiency. Using the concept of Fourier heat conduction and thermal to electrical analogy, the electrical resistance and heat conductance of the thermocouple materials, $A$ and $B$, are expressed as [20]:

$$
\begin{aligned}
& R_{e}=\frac{L_{A}}{A_{A} \sigma_{A}}+\frac{L_{B}}{A_{B} \sigma_{B}} \\
& K=\frac{A_{A} k_{A}}{L_{A}}+\frac{A_{B} k_{B}}{L_{B}}
\end{aligned}
$$

Where $A$ equals the cross sectional area, $\mathrm{cm}^{3}, L$ equals arm length, $\mathrm{cm}, \sigma$ equals electrical conductivity, $1 / \Omega . c m$ of the module. The current through the load per thermocouple is:

$I=\frac{V_{L}}{R+R_{L}}=\frac{\alpha\left(T_{h}-T_{c}\right)}{R+R_{L}}$

From equation (9), the power delivered is expressed as:

$$
P=\frac{\alpha^{2}\left(T_{h}-T_{c}\right)^{2}}{\left(R+R_{L}\right)^{2}} \times R_{L}
$$

In the light of equation (16) and equation (29), the efficiency of the thermoelectric generator can be expressed as:

$$
\eta=\frac{T_{h}-T_{c}}{T_{h}} \times\left[\left(\frac{R+R_{L}}{R_{L}}\right)^{2} \cdot \frac{K}{\propto^{2} T_{h}}+\frac{R}{2 R_{L}}+\frac{R T_{c}}{2 R_{L} T_{h}}+1\right]^{-1}
$$

Substituting equation (4) into equation (30), the expression for the efficiency becomes:

$\eta=\eta_{c}\left[1+\frac{1}{2 m}\left(1+\frac{T_{c}}{T_{h}}\right)+\frac{(m+1)^{2}}{m} \cdot \frac{1}{T_{h} Z}\right]^{-1}$

Where, $Z$, is the figure of merit of the thermocouple expressed as:

$Z=\frac{\propto^{2}}{K R}$

The dimension of $Z$ and its unit of measure in $\mathrm{SI}$ is $K^{-1}$. For higher efficiency the product, $K R$, must be kept to minimum and this minimum occurs when the length and cross sectional area satisfy the relation:

$\frac{L_{A} A_{B}}{L_{B} A_{A}}=\sqrt{\frac{k_{A} \sigma_{A}}{k_{B} \sigma_{B}}}$

Using the conditions defined by equation (33), $K R$ product is:

$K R=\left[\left(\frac{k_{A}}{\sigma_{A}}\right)^{1 / 2}+\left(\frac{k_{B}}{\sigma_{B}}\right)^{1 / 2}\right]^{2}$

For maximum efficiency,

$\frac{d \eta^{*}}{d m}=0$

$\frac{d}{d m}\left[\left(\frac{m+1}{m}\right)^{2} \cdot \frac{1}{z T_{h}}+\frac{1}{2 m}\left(1+\frac{T_{c}}{T_{h}}\right)+1\right]^{-1}=0$

Upon differentiation of equation (36) and simplification:

$$
\begin{gathered}
m=\sqrt{1+Z T_{m}} \\
\text { Where: }
\end{gathered}
$$

$T_{m}=\frac{T_{h}-T_{c}}{2}$

Substituting equation (37) into the expression for equation (31) yields:

$$
\eta^{*}=\frac{\sqrt{1+Z T_{m}}-1}{\sqrt{1+Z T_{m}}+\frac{T_{c}}{T_{h}}}=\frac{m-1}{m+\frac{T_{c}}{T_{h}}}
$$

And the optimum generator efficiency is thus obtained as:

$\eta_{\text {opt }}=\frac{T_{h}-T_{c}}{T_{h}} \times \frac{m-1}{m+T_{c} / T_{h}}$

\section{Stepper Motor Drive Mechanism}

In order to save the thermoelectric modules from excessive temperatures and to prevent thermally induced failure, the flame temperature is regulated by causing a differential gas flow rates by opening and or closing the gas valve using a microcontrolled stepper motor. The stepper motor driver is basically a ULN 2003 Darlington transistor array. Figure 1 shows the electrical connection of the stepper motor driver. 
The ULN 2003 chip has 7 inputs and 7 output, with a total of 200 steps per revolution, implying a degree per step of $1.8^{\circ}$. Table 1 shows the rotation of the stepper motor through a single step in the clockwise direction. Where 0 represents 0 volts and 1 represents 5 volts. By sending this sequence of pulses from the micro-controller $(\mu C)$, the stepper motor will move a step in the clockwise direction. Table 2 shows the rotation of the stepper motor through a single step in the anticlockwise direction.

By reversing the direction of the pulses, the stepper motor moves one step in the anticlockwise direction. The pulse sequences are repeated quickly to make the stepper motor rotate. Given the number of pole pairs, $n$, and stator phases, $m_{p}$, the steps per revolution and step angles are obtained from equation (37) and equation (38) respectively [21]:

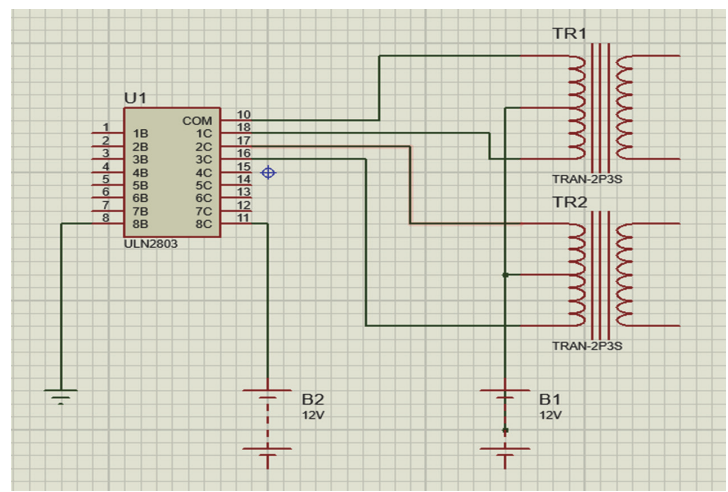

Figure 1 Electrical arrangement of a stepper motor driver

Table 1 Single step rotation of the stepper in the clockwise direction

\begin{tabular}{|c|c|c|c|}
\hline$\mu C_{1}$ & $\mu C_{2}$ & $\mu C_{3}$ & $\mu C_{4}$ \\
\hline 1 & 0 & 0 & 0 \\
\hline 0 & 1 & 0 & 0 \\
\hline 0 & 0 & 1 & 0 \\
\hline 0 & 0 & 0 & 1 \\
\hline
\end{tabular}

Table 2 Single step rotation of the stepper in the anticlockwise direction

\begin{tabular}{|c|c|c|c|}
\hline$\mu C_{1}$ & $\mu C_{2}$ & $\mu C_{3}$ & $\mu C_{4}$ \\
\hline 1 & 0 & 0 & 0 \\
\hline 0 & 0 & 0 & 1 \\
\hline 0 & 0 & 1 & 0 \\
\hline 0 & 1 & 0 & 0 \\
\hline
\end{tabular}

$S=2 n m_{p}$

$\Delta \phi=\frac{360}{S}$

The total torque produce by the stepper motor is:

$$
T_{M}=\sum_{j=I}^{m} T_{M j}
$$

Where the phase motor torque is expressed as:

$$
T_{M j}=k_{m} \sin \left[n \phi(t)+\phi_{0 j}\right] \cdot I_{j}(t)
$$

The current $I_{j}(\mathrm{t})$ in the coil is a function of the supplied voltage $U_{j}$ and the coil properties and are related by the expression:

$$
U_{j}=e m f_{j}+R \cdot I(t)+L \frac{d I(t)}{d t}
$$

The emf in each coil can be expressed as:

$$
e m f_{j}=k_{m} \sin \left[n \phi(t)+\phi_{0 j}\right] \cdot \omega
$$

The total load acceleration torque is:

$$
T_{A}=\sum_{j=1}^{m} T_{M j}-D \omega-T_{F}
$$

Where $D$ equals the viscous damping constant, $\mathrm{Nm} / \mathrm{s}, \omega$ is the rotational speed, $\mathrm{rad} / \mathrm{s}$ and $T_{F}$ is the frictional torque, $N$. The actual rotor position $\phi_{i}, \mathrm{rad}$, is:

$\phi_{i}=(x-y) \cdot \Delta \phi$

The commanded position $\phi_{i+1}$, will then be [21]: $\phi_{i+1}=\phi_{i} \pm \Delta \phi$

Where (+) stands for positive, (-) for negative voltages. Table 3 shows the pulses for both phases required for 1 revolution.

Table 3 Pulses for both Phases required for 1 Revolution

\begin{tabular}{|l|l|l|}
\hline Rotor position & Voltage in phase 1 & Voltage in phase 2 \\
\hline $0 / 360^{\circ}$ & 0 & $-U_{2}$ \\
\hline $90^{\circ}$ & $U_{1}$ & 0 \\
\hline $180^{\circ}$ & 0 & $U_{2}$ \\
\hline $270^{\circ}$ & $-U_{2}$ & 0 \\
\hline
\end{tabular}

The required pulse for each phase with a given $\phi_{i+1}$ may be calculated by: 


$$
P_{j}=\sin \left(\phi_{i+1} n+\phi_{0}\right)
$$

The voltage sensor is a simple voltage divider arrangement of resistors as shown in Figure 2.

The measured voltage is defined by the relation:

$V_{\text {in }}=V_{\mu c u} \times \frac{R_{1}+R_{2}}{R_{2}}$

$V_{\mu c u}$ is the voltage seen by the micro-controller. The micro-controller measures $V_{\mu c u}$ and then interpolates in the program to know the amount of voltage $V_{\text {in }}$ being measured. $R_{1}$ was chosen as 2200 $\Omega$ while $R_{2}$ was chosen as $10 \mathrm{k} \Omega$. The computer interface is a visual basic program that communicates with the micro-controller via a serial port connection. Commands are sent from the interface to the micro-controller and it respond with all the measured data-values. These values are then displayed on the user-interface in a graphical mode and as texts. The ATMEGA32 being the heart of the system, controls the system activities with the help of the program codes embedded in it. The programming language for the ATMEGA32 source code is C, due to its ease of programming when compared to assembly language which is compiled to have the hex object code to be used by the microcontroller.

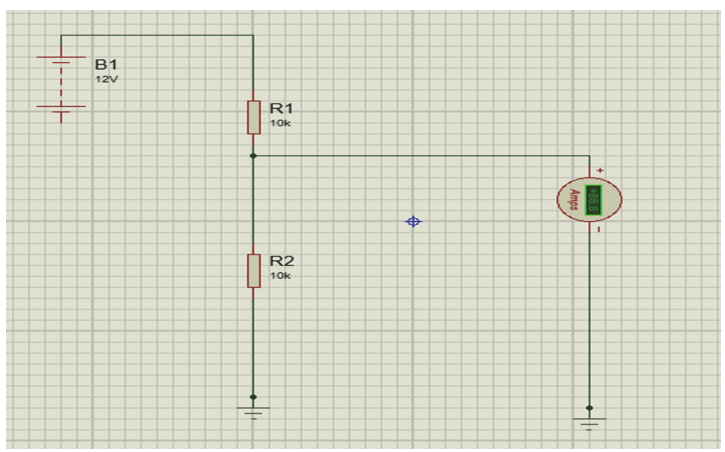

Figure 2 Voltage Sensor

\section{Methodology}

The performance matrix models of a thermoelectric generator were modelled and solved using MATLAB. The thermoelectric generator was developed using ten SP-184827145-SA thermoelectric modules tested for the purpose of validation. The experimental setup consists of the thermoelectric modules, a water cooled heat sink, propane gas burner heat source, temperature control circuitry, storage batteries and a power inverter. Figure 3 shows the modules attached to the heat sink with the aid of thermal paste, a base plate is bolted to the heat sink with the modules sandwiched between them to give the generator assembly in figure 4 .

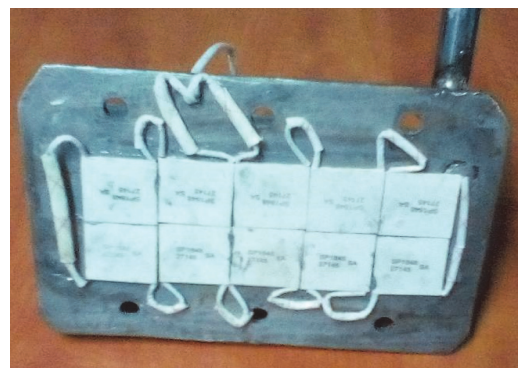

Figure 3 Heat Sink and Modules Assembly

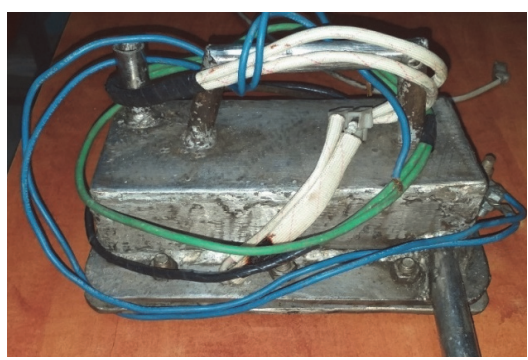

Figure 4 Thermoelectric Generator

(a)

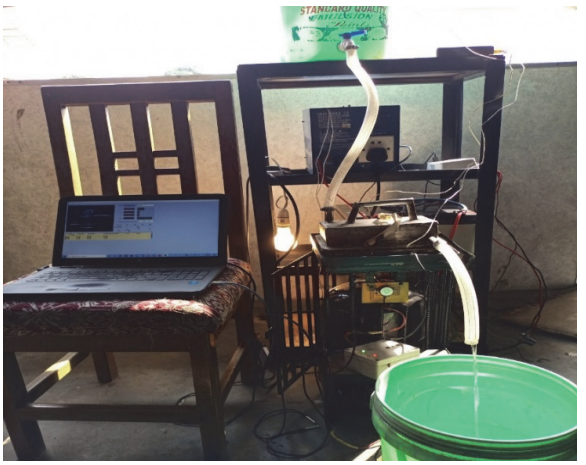

(b)

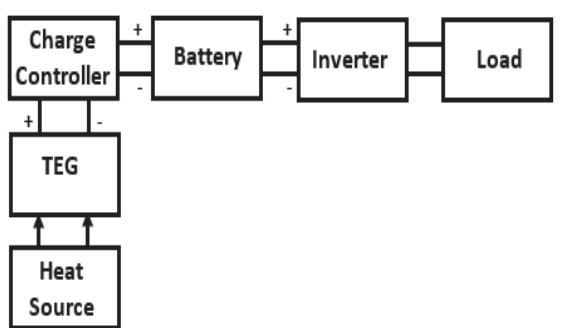

Figure 5 Experimental Setup: a. Test Rig; b. Wiring Diagram

The heat source to the generator is combustion of propane gas, due to the high temperatures of combustion and need to safeguard the modules from thermally induced failure, the flame temperature was controlled. The temperature control cir- 
cuitry consists of an ATMEGA32 microcontroller, DS18B30 temperature sensors, stepper motor, computer interface and power supply unit. Figure 5 shows the experimental setup used for data collection.

While figure 6 on the other hand shows the control flow chat.

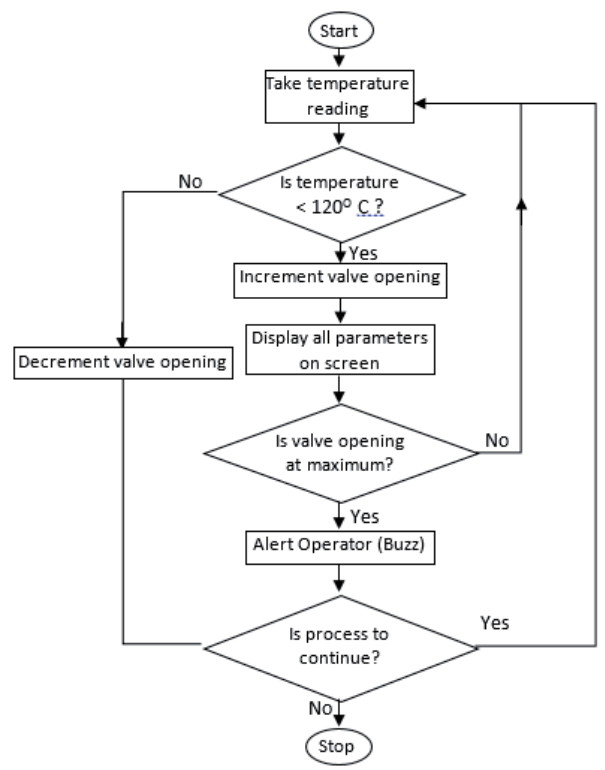

Figure 6 Control flow chat

\section{Results and Discussion}

\subsection{Simulation of Voltage with Hot Junction Temperature}

Figure 7 shows the simulated variation of open circuit voltage with hot junction temperature at different cold side temperatures. The generated voltage from the thermoelectric generator increases linearly with an increase in hot side temperature and decreases with an increase in cold side temperature. Rohit et al [6] obtained similar result, implying that the more the temperature gradient the more the voltage generated.

\subsection{Simulation of Current against Hot Junction Temperature}

Figure 8 show the simulated plots of short circuit current against hot side temperature at various cold side temperatures. The behaviour of the current plots follows a similar profile as that of the voltage plots, this is however expected due to the linearity of Ohm's law. Note that a singular advantage of this trend is the higher the current output the more the load that can be catered to by the generator. An increase in hot side temperature leads to an increase in the current output and an increase in cold side temperature leads to a decrease in current output. Thus for maximize the output current, the hot junction temperature should be kept as high as possible and the cold junction temperature should be kept as low as possible, which can be verified using the research of Elzalik et al. [22].

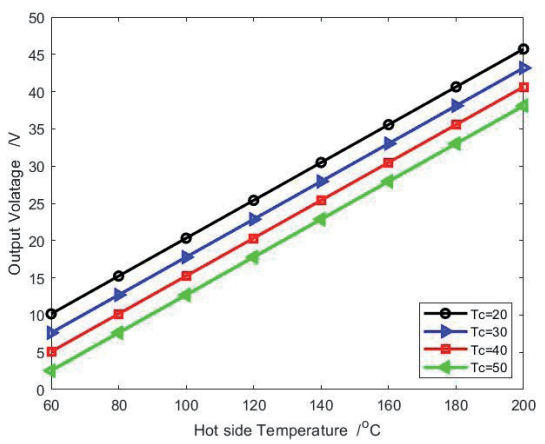

Figure 7 Voltage vs Hot junction temperature

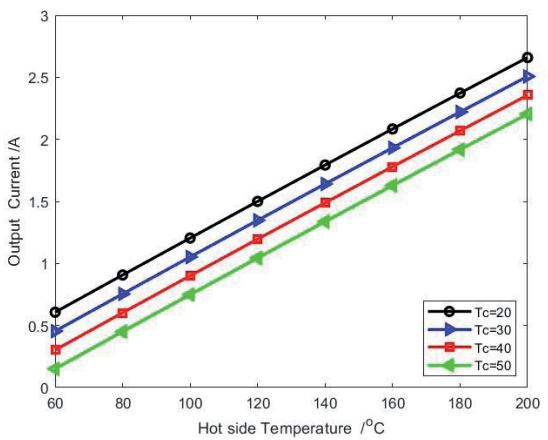

Figure 8 Current vs Hot Side Temperature

\subsection{Simulation of Power against Hot Side Temperature}

Figure 9 illustrates the simulated variation of power output with different hot junction temperatures and at various cold side temperatures. Clearly the power output increases with an increase in hot side temperature and decreases with an increase in cold side temperature. Similar result was obtained by Niu et al [23] and Rowe and Min [24], another testimony to the fact that as temperature gradient increases the power output surges.

\subsection{Simulation of Efficiency against Hot Side Temperature}

Figure 10 is a simulated generated plots of efficiency against hot side temperatures at varied cold side temperatures. Efficiency can be seen to increase with an increase in hot side temperature and decreases with an increase in cold side temperature, thus the greater the temperature gradient the more the efficiency of conversion. 


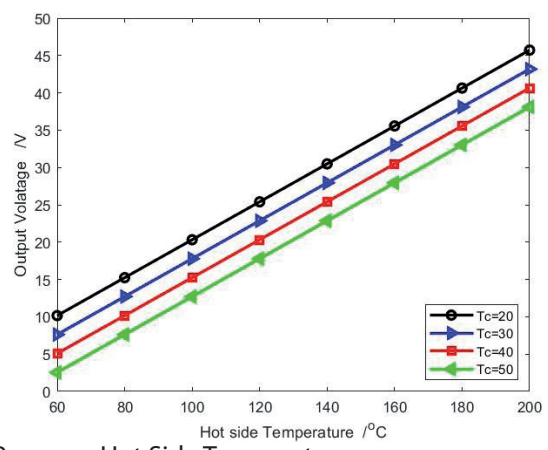

Fig. 9 Power vs Hot Side Temperature

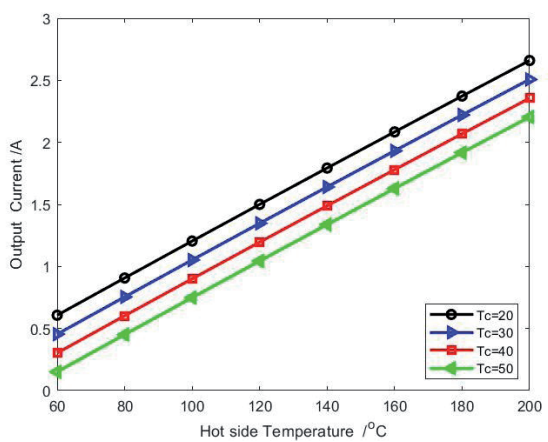

Fig. 10 Efficiency vs Hot Side Temperature

\subsection{Simulation of Laminar Flame Velocity with Initial Gas Temperature}

Figure 11 show simulated plots of laminar flame velocity against initial gas temperature of propane gas.

The flame velocity increases slightly with an increase in initial gas temperature increases markedly with an increase in mixture strength. Ebaid and AlKhishali [25] obtained similar results for propane air mixture. A 3.32\% increase in initial gas temperature resulted in $8.45 \%$ increase in laminar flame speed while a $33.33 \%$ increase in mixture strength resulted in a $444.4 \%$ increase in laminar flame velocity.

\subsection{Angular Rotation and Percentage Opening of Gas Valve against stepper motor Steps}

Figure 12 shows the graph of angular rotation and percentage opening of gas valve against steps of stepper motor. The stepper motor has 200 steps with $1.8^{\circ}$ rotation per step. The minimum number of steps required to ignite flame is 20 steps which is equivalent to $36^{\circ}$ rotation. Clearly for every increase in steps of the stepper motor there is an equivalent increase in angular rotation of the gas valve and a corresponding increase in percentage opening of gas valve. This results in increase in flame produce and thus, increase in temperature. It can be ob- served that after the 100th step, there is greater increase in percentage opening of the gas valve. The microcontroller system was programmed to close the gas valve at $120^{\circ} \mathrm{C}$ to protect the thermoelectric modules from thermal induced failure, ensuring no slip between the stepper motor gas valve interface.

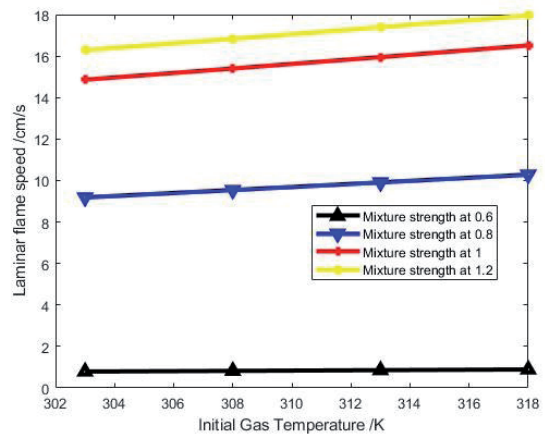

Fig. 11 Laminar Flame Speed vs Initial Gas Temperature

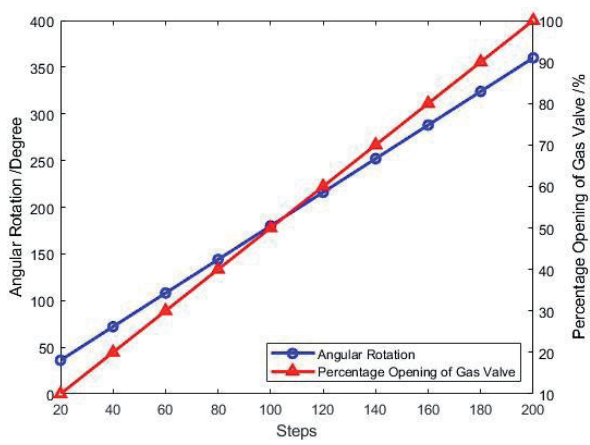

Fig. 12 Angular Rotation and Percent Gas Valve opening vs Stepper Motor Steps

\subsection{Experimentation}

5.7.1 Experimental Hot and Cold Side Temperature with Time

Figure 13 shows the plot of hot and cold side temperature against time as obtained from ATMEGA32 microcontroller. Clearly the microcontroller was able to regulate the gas valves to the set value of $120^{\circ} \mathrm{C}$ to protect the modules from excessive temperature and damage due to thermally induced failure while cold side temperature is almost constant during the course of the experiment.

The module hot side temperature increases greatly as time increases though wavy due to instability of the flame speed. The microcontroller as can be seen was able to regulate the gas flow rate for combustion thereby safeguarding the modules.

5.7.2 Experimental and theoretical voltage against temperature difference

Figure 14 shows the graph of experimental and theoretical open circuit voltage against temperature 
difference.

Clearly the experimental and theoretical voltages follow identical profile. The theoretical voltage shows a linear relationship while the experimental voltage moved in curve like manner which is due the unsteady state of the flame speed occasion by the operation of the microcontroller in regulating the gas flow rates.

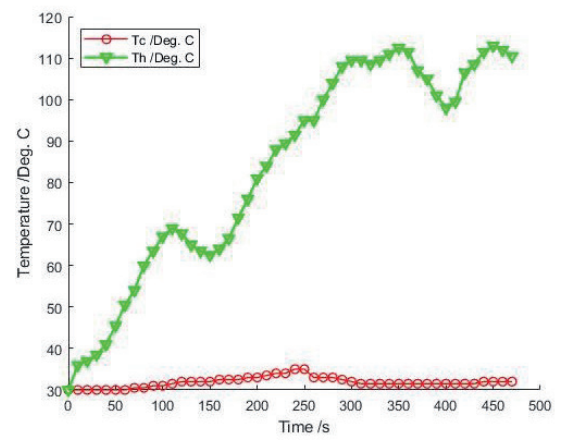

Fig. 13 Hot and Cold side Module Surface Temperature vs Time

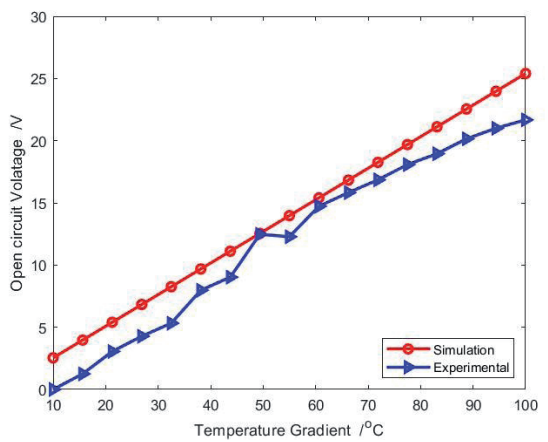

Fig. 14 Experimental and theoretical voltage vs temperature difference

\section{Conclusion}

Thermoelectric generators are regarded as solidstate devices as they implore the use of Seebeck effect in its operation by taking advantage of temperature difference to create electrical energy, they can serve as an alternative source of power supply for off grid locations. Hence this research successfully modelled, simulated and experimented a prototype thermoelectric generator whose input source of energy is combust propane gas with inbuilt temperature regulation mechanism to protect the modules from excessive temperatures likely to induce thermal failures. Results showed that the simulated and experimented open circuit voltages are in good correlation with a maximum experimented voltage of $25 \mathrm{~V}$ with hot side temperature of $120^{\circ} \mathrm{C}$ and cold side temperature of $30^{\circ} \mathrm{C}$ and an efficiency of $3.6 \%$. Thus a thermoelectric generator serves as a reliable source of electricity as it contains no moving parts subject to wear and tear like in rotodynamic machineries.

\section{References}

1. Ismail B. I., Ahmed W. H. (2009). Thermoelectric power generation using waste-heat energy as an alternative green technology. Recent Patents on Electrical and Electronic Engineering (Formerly Recent Patents on Electrical Engineering), 2(1), pp. 27-39.

2. Wu Y., Ming T., Li X., Pan T., Peng K., Luo X. (2014). Numerical simulations on the temperature gradient and thermal stress of a thermoelectric power generator. Energy conversion and management, 88, pp. 915-927.

3. Singh T., Marsh R., Min G. (2016). Development and investigation of a non-catalytic self-aspirating meso-scale premixed burner integrated thermoelectric power generator. Energy Conversion and Management, 117, pp. 431-441.

4. Kim K. J. (2010). Thermal and power generating performances of thermoelectric energy recovery modules. 12th IEEE Intersociety Conference on Thermal and Thermomechanical Phenomena in Electronic Systems, pp. 1-7.

5. Shah P., Babu D., Deshpande V. (2015). Analysis of Power generation through thermoelectric generators. International Journal of latest trends in engineering and technology, 6( 2), pp. 110-118.

6. Rohit G., Manaswini D., Kotebavi V., Nagaraja S. (2017). Performance study of thermo-electric generator. AIP Conference Proceedings 1859, 020094

7. Ming T., Wang Q., Peng K., Cai Z., Yang,W., Wu Y., Gong T. (2015). The influence of non-uniform high heat flux on thermal stress of thermoelectric power generator. Energies, 8(11), pp. 12584 12602.

8. Ahiska R., Dişlitaş, S. (2006). Microcontroller based thermoelectric generator application. Gazi University urnal of Science, 19(2), pp. 135-141.

9. Nuwayhid R. Y., Shihadeh, A., Ghaddar N. (2005). Development and testing of a domestic woodstove thermoelectric generator with natural convection cooling. Energy Conversion and Management, 46(9-10), pp. 1631-1643.

10. Chasmar R. P., Stratton R. (1959). The thermoelectric figure of merit and its relation to thermoelectric generators. International journal of electronics, 7(1), pp. 52-72.

11. Mishra A. K., Singh A. K, Prakash A., Ambekar R. S. (2017). Thermoelectric Generator. International Research Journal of Engineering and Technology (IRJET), 4(6), pp. 854-856.

12. Elghool A., Basrawi F., Ibrahim T. K., Habib K., Ibrahim H., Idris 
D. M. N. D. (2017). A review on heat sink for thermo-electric power generation: Classifications and parameters affecting performance. Energy conversion and management, 134, pp. 260-277.

13. Lee J., Kim S., Marconnet A., Zandt M. A. A.in't., Asheghi M., Wong H. S. P., Goodson K. E. (2012). Thermoelectric characterization and power generation using a silicon-on-insulator substrate. Journal of Microelectromechanical Systems, 21(1), pp. 4-6.

14. AravindKaruppaiah C., Ganesh. S., Dileepan T., Jayabharathi S. (2014). Fabrication and Analysis of Thermoelectric Generator for Power Generator. International Journal of Innovative Research in Science, Engineering and Technology, 3(1), pp. 15081513.

15. Jfri F., Tawil S. N. M., Syaripuddin M., Mohamad T. N. T., Miskon A. (2015). Employment of waste heat for thermoelectric-based energy harvesting. ARPN Journal of Engineering and Applied Sciences, 10(20), pp. 9896-9901.

16. Lay-Ekuakille A., Vendramin G., Trotta A., Mazzotta G., (2009). Thermoelectric generator design based on power from body heat for biomedical autonomous devices, IEEE International Workshop on Medical Measurements and Applications, Cetraro, Italy.

17. Stecanella P. A. J., Faria M. A. A., Domingues E. G., Gomes P. H. G., Calixto W. P., Alves A. J. (2015). Electricity generation using thermoelectric generator-TEG. IEEE 15th International Conference on Environment and Electrical Engineering, pp. 2104- 2108).

18. Rowe D. M. (1978). Thermoelectric power generation. Proceedings of the Institution of Electrical Engineers, Vol. 125, No. 11 R, pp. 1113-1136.

19. Metghalchl M., Keck J. C. (1982). Burning Velocities of Mixtures of Air with Methanol, Isooctane, and Indolene at High Pressure and Temperature. Combustion and Flame, 48, pp. 191-210.

20. Lee H. (2010). Thermal Designs: Heat Sinks, Thermoelectrics, Heat Pipes, Compact Heat Exchangers and Solar cells. John Wiley and Sons. Inc., pp.105-113.

21. Morar, A. (2003). Stepper Motor Model for Dynamic Simulation. Acta Electrotehnica, 44(2), pp. 117-122.

22. Elzalik M., Rezk H., Mostafa R., Thomas J., Shehata E. G. (2019). "An Experimental Investigation On Electrical Performance and Characterization of Thermoelectric Generators". International Journal of Energy Research. DOI: 10.1002/er.4873, pp 1-16.

23. Niu X., Yu J., Wang S. (2009). Experimental Study on Low Temperature Waste Heat Thermoelectric Generator. Journal of Power Sources, 188, pp. 621-626.

24. Rowe D. M., Min G. (1998). Evaluation of thermoelectric modules for power generation. Journal of power sources, 73(2), pp. 193-198.

25. Ebaid M. S., Al-Khishali K. J. (2016). Measurement of the Laminar Burning Velocity for Propane: Air mixtures. Advances in
Mechanical Engineering, 8(6), pp. 1-17.
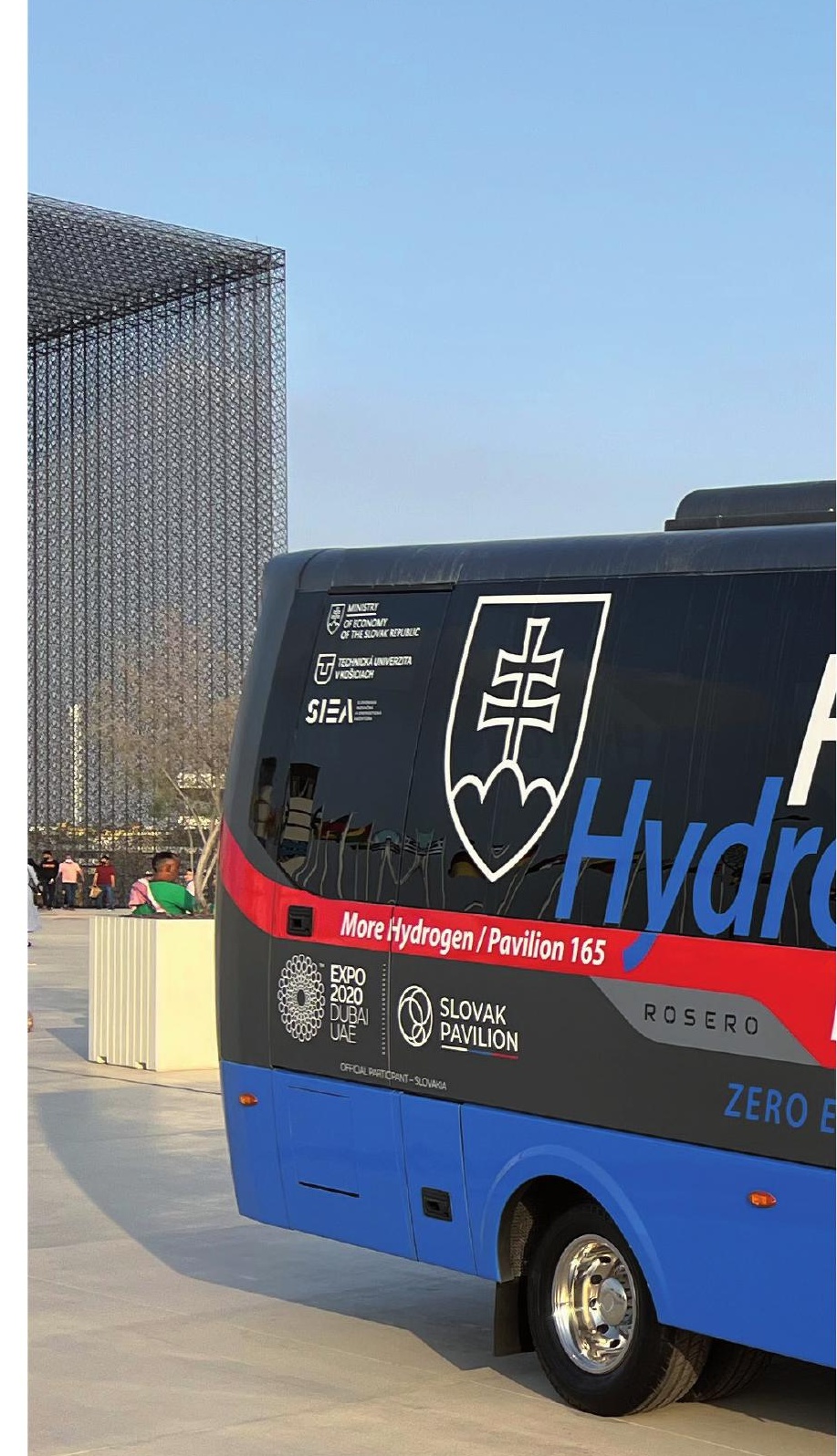